\title{
NEURAL NETWORK SYSTEMS FOR ESTIMATING THE INITIAL CONDITION IN A HEAT CONDUCTION PROBLEM
}

\author{
Elcio Hideiti Shiguemori ${ }^{1}$, José Demisio Simões da Silva ${ }^{1,2}$, \\ Haroldo F: Campos-Velho ${ }^{1}$ \\ ${ }^{1}$ Instituto Nacional de Pesquisas Espaciais - LAC-INPE \\ Av. dos Astronautas, 1758, J. Granja, São José dos Campos, SP, Brazil \\ ${ }^{2}$ Universidade Braz Cubas - UBC \\ Av. Francisco Rodrigues Filho, 1233, Mogi das Cruzes, SP, Brazil \\ Emails: \{elcio, demisio, haroldo\}@ lac.inpe.br
}

\begin{abstract}
This paper describes a neural network approach to the inverse problem of determining the initial temperature distribution on a slab with adiabatic boundary conditions, from transient temperature distribution, obtained at a given time. Two neural network architectures have been proposed to address the problem: the multilayer perceptron with backpropagation and radial basis functions (RBF), both trained with the whole temperature history mapping. The conducted simulations showed RBF networks present better solutions, faster training, but higher noise sensitiveness, as compared to the multilayer perceptron with backpropagation.
\end{abstract}

\section{Introduction}

A direct (forward) problem solution consists of finding effects from a complete description of its causes. On the other hand, in solving an inverse problem, one is interested in determining unknown causes from observed or desired effects. Different kind of inverse problems can be found in the literature, based on the nature of estimated property [13]: estimation of initial/boundary conditions, properties of the system/material, sources or sink terms, shape and governing equations.

There exist some works in the literature that describe the use of neural networks employed as tools to solve inverse problems [3][8][14][15][16] in applications such as heat transfer and electromagnetics.

This paper describes a neural network based approach to determine the initial temperature distribution on a slab with adiabatic boundary conditions, from a transient temperature distribution, obtained at a given time. In [4], this heat transfer problem is considered to be an ill-posed inverse problem, where the initial condition has to be estimated.

Two NNs models have been used: a multilayer perceptron neural network with backpropagation and Radial Basis Function (RBF) networks

The simulations with the proposed models show the effectiveness for solving the inverse problem at hand, under the problem approach of using the whole temperature history mapping (WHM) for training purposes [3]. Comparisons of the simulations with both models have shown a better performance of the RBF network with Gaussian functions, because of faster training and better solutions to the problem, but with higher sensitiveness to noise as compared to multilayer percetpron with backpropagation.

In section 2 the inverse problem is presented. Section 3 describes the neural network architectures used. Section 4 shows implementation details and section 5 comments on the simulation results. Section 6 makes some conclusions.

\section{The Inverse Problem}

The direct problem consists of a transient heat conduction problem in a slab with adiabatic boundary condition, initially at a temperature denoted by $f(x)$, given by the heat equation (1):

$$
\begin{aligned}
& \frac{\partial^{2} T(x, t)}{\partial x^{2}}=\frac{\partial T(x, t)}{\partial t}, \text { for }(x, t) \in \Omega \times \mathrm{R}^{+}, \\
& \frac{\partial T(x, t)}{\partial x}=0, \quad(x, t) \in \partial \Omega \times \mathrm{R}^{+}, \\
& T(x, 0)=f(x), \quad(x, t) \in \Omega \times\{0\}
\end{aligned}
$$

In (1), $T(x, t)$ is the temperature, $f(x)$ is the initial condition, $x$ is the spatial variable, and $t$ is time. All of these variables are dimensionless quantities and $\Omega=[0,1]$.

There exists a solution to the direct problem for a given initial condition $f(x)$ that is explicitly obtained using separation of variables [4], for $(x, t) \in \Omega \times \mathrm{R}^{+}$:

$T(x, t)=\left.\sum_{m=0}^{+\infty} e^{-\beta_{m}^{2}} \frac{1}{N\left(\beta_{m}\right)} X\left(\beta_{m}, x\right)\right|_{0} ^{1} X\left(\beta_{m}, \dot{x}\right) f(\dot{x}) d \dot{x}$

where $X\left(\beta_{m}, x\right)=\cos \left(\beta_{m} x\right)$ are the eigenfunctions associated to the problem, $\beta_{m}=m \pi$ the eigenvalues and $N\left(\beta_{m}\right)=$ $\int_{\Omega} X\left(\beta_{m}, x^{\prime}\right) f\left(x^{\prime}\right) d x^{\prime}$ represents the integral normalization (or the norm).

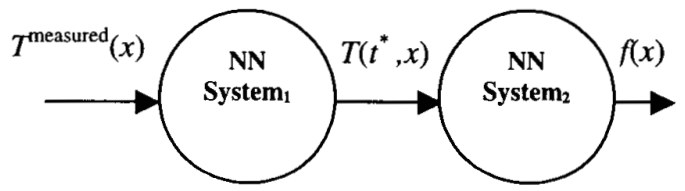

Figure 1-Block Diagram of the NN approach. 
In this paper, the inverse solution is obtained using a neural network approach, as it is shown in the diagram in Figure 1.

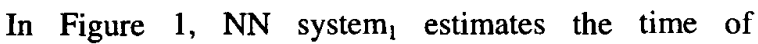
occurrence of the transient temperature distribution and $\mathrm{NN}$ system $_{2}$ estimates the initial condition, that is, the temperature distribution at time $t=0$. Both $\mathrm{NN}$ systems are implemented using multilayer perceptrons in one experiment, and RBF networks in a second experiment.

\section{Backpropagation and RBF Neural Networks}

Neural networks have become important tools for information processing [10] and [1]. Much research has been conducted in pursuing new neural network models and adapting the existing ones to solve real life problems, such as those in engineering [10] and [1]. All the applications developed make use of the characteristics NNs present, such as:

- A large number of simple neuronlike processing elements.

- A large number of weighted connections among the elements that encode the knowledge in a network.

- Highly parallel distributed control.

- An emphasis on automatic learning of internal representations.

The arrangements of these neuronlike elements, result in massively parallel networks of simple elements that can achieve a solution at a very high speed and, and at the same time, display insensitivity to the loss and the failure of a number of component elements in the network [12]. These properties have made neural networks appropriate for applications in pattern recognition, signal processing, image processing, computer vision, engineering, etc. [1][9][10] [12].

Multilayer perceptrons with the backpropagation learning algorithm are the most used neural network architectures, with application in several different areas. They are basically composed of an input layer, an output layer, and a number of hidden layers that extract high order statistics from the input data [12]. A supervised learning algorithm controls the training phase. Then, the input and output (desired) data need to be provided, which permit the calculation of the error of the network. The network's weights adjustment is conducted by backpropagating such error to the network. The weights are changed by an amount proportional to the error at that unit times the output of the unit feeding into the weight. Equation (3) shows the general weight correction (the delta rule).

$$
\Delta w_{j i}=\eta \delta_{j} y_{i}
$$

$\delta_{j}$ is the local gradient of neuron $j, y_{i}$ is the input signal of neuron $j$, and $\eta$ is the learning rate parameter that controls the strength of change.
Radial basis function networks are feedforward networks with one hidden layer, developed for data interpolation in multidimensional space [7]. RBFs can be used to learn arbitrary mappings and differ from multilayer perceptrons with backpropagation in that the RBF hidden layer units have a receptive field with a center, through which a particular input value has a maximal output. Their output tails off as the input moves away from this point. Generally, the RBF hidden unit function is a Gaussian function (Figure 2) with null mean and standard deviation $\sigma^{2}$, though other Gaussian like functions may be used [11].

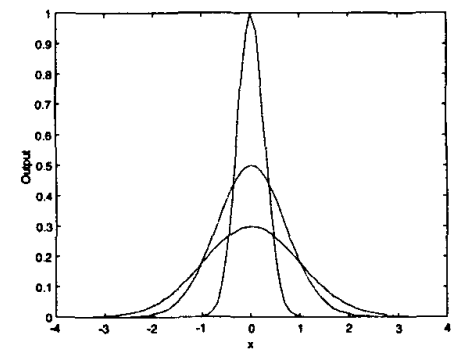

Figure 2 - Gaussians with three different standard deviations.

In training RBF networks, decisions have to be made on the number of hidden units, the centers, the sharpness (standard deviation) of the Gaussians, and the output layer training. The centers and standard deviations are generally decided on first by examining the vectors in the training data [6]. The Delta Rule [2][5][11] is used to train the weights in the output.

RBF networks are used for classification problems and function approximation. They have the advantage that one can add extra units with centers near parts of the input, which are difficult to classify.

Both Backpropagation and RBFs networks can be used for processing time-varying data and many other applications.

\section{Implementation}

The approach adopted for the inverse problem in this paper is based on the Whole History Mapping (WHM) [3]. A $\mathrm{NN}$ is designed to map the whole vector of observed values of the temperature history to the corresponding output vector [3]. Thus, the training process leads to a correspondence between the observed temperature values and the output, over some time interval.

The WHM has the advantage of being quite stable and insensitive to noise in the data [1]. However, two critical disadvantages are: 1) a large number of input vectors may result in a large number of connections, which can imply very slow training; and 2) for every new case, the network has to be retrained with the new training set. 


\section{Neural Network Architectures}

The performances of the different NN Systems are compared in estimating the initial temperature condition. Two neural network systems are proposed: a 2-multilayer perceptron neural network system with backpropagation and a 2-RBF neural network system.

Figure 5 presents the activation process of both systems after training. In each $\mathrm{NN}$ system, network 1 estimates the time of occurrence of a given transient temperature distribution (only 1 output neuron is required), and network 2 estimates the initial temperature distribution over the slab (the $n$ output neurons represent different positions over the slab) by iterating its output, down to time $t=0$ from the estimated time in network 1.

In this paper, results are shown for two experiments each of which having two NN, as described previously. Following are the NN architectures used in both experiments:

\section{NN System 1:}

- 2 Backpropagation networks:

- 1 input layer - 1 hidden layer - 1 output layer

- Sigmoidal hidden neurons

- Sigmoidal output neurons

NN System 2:

- 2 RBF networks:

- 1 input layer - 1 hidden layer - 1 output layer

- Gaussians Radial basis neurons

- Linear output neurons Linear

\section{Training Data}

The training set is composed of three temperature distributions obtained by applying the direct model of [4] (equation 2), considering a slab with adiabatic conditions (Figure 3 ) on which 25 reading sensors are placed (along the slab length). The three different distributions used (triangular, logarithmic, and semi sinusoidal) are developed for 50 time slices and appended as in Figure 3, to form the training data.

Two different training sets are extracted: one for training network 1; and the other for training network 2 that estimates the initial condition (Figure 4).

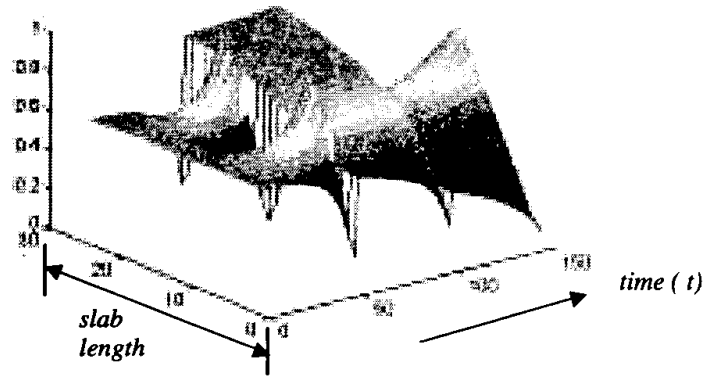

Figure 3 - Appended temperature distributions over 25 positions on the slab, for 50 time slices.
The input data for network 1 is the temperature distribution history (Figure 4-a left) and the target data is the time of occurrence of the each temperature distribution over the slab (Figure 4-a right). For network 2 the input is the time appended temperature distribution (Figure 4-b left) and the target data is the shifted temperature distribution (Figure 4-b right).
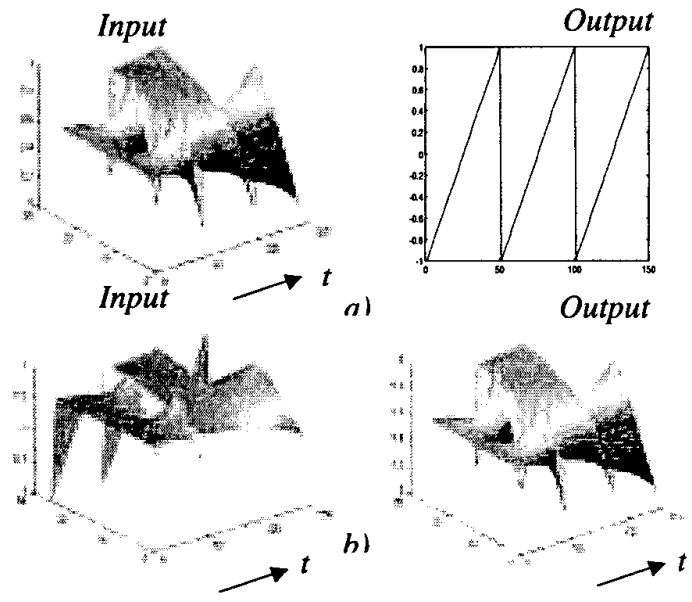

Figure 4 - Training sets used. a) Input and Output data set for network 1; b) Input and Output data set for network 2.

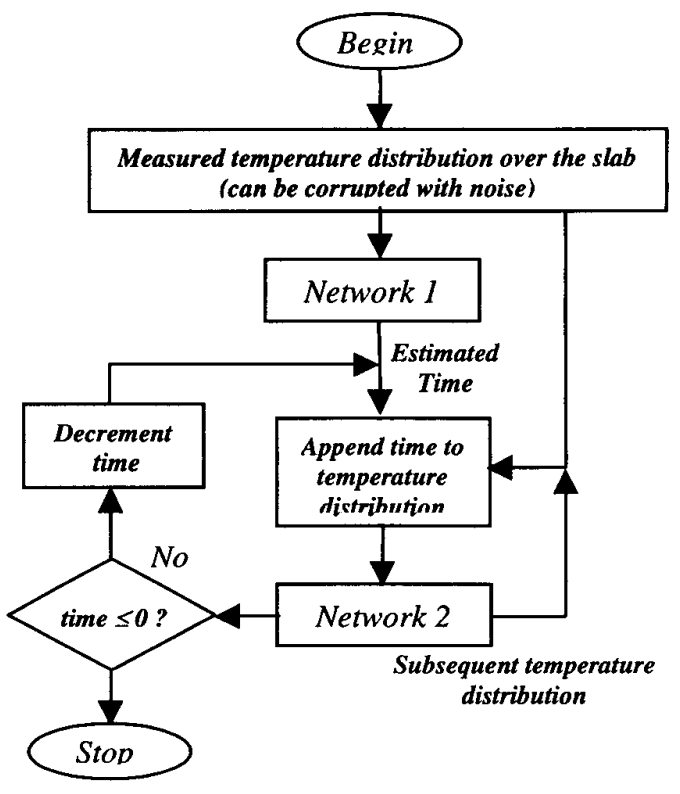

Figure 5 - Flowchart of the NN activation in the general approach for solving the inverse problem described in [4].

In either experiment, the training of network 2 consists in presenting a measured temperature profile with its corresponding time slice $(t)$ at the input, and the following distribution at time $(t+1)$ as the output. Thus, network 2 is trained to estimate the distribution at a time step ahead, 
whereas network 1 estimates the time of occurrence of the measured transient temperature distribution supplied.

Table 1 - Training results for the multilayer perceptron neural network with backpropagation.

\begin{tabular}{|c|c|c|c|}
\hline network & $\begin{array}{c}\text { \# of } \\
\text { neurons }\end{array}$ & $\begin{array}{c}\text { Target } \\
\text { error }\end{array}$ & $\begin{array}{c}\text { \# of } \\
\text { epochs }\end{array}$ \\
\hline 1 & 50 & 0.001 & 20000 \\
\hline 2 & 50 & 0.0001 & 20000 \\
\hline
\end{tabular}

The networks are trained separately in either experiment. Table 1 shows the training results for the multilayer perceptron neural network with backpropagation. Both networks are trained up to the stopping criteria. Network 1 achieved the target error, whereas network 2 was trained for the maximum number of epochs.

The training results for the RBF networks are shown in table 2. The algorithm for training the RBF networks gradually builds up the final network, thus not requiring the specification of the number of neurons in the hidden layer.

In both simulations, the training parameters are chosen by testing different possibilities and checking out the networks' performances.

Table 2 - Training results for the RBF networks.

\begin{tabular}{|c|c|c|c|c|}
\hline network & $\begin{array}{c}\text { RBF } \\
\text { function }\end{array}$ & $\begin{array}{c}\text { \# of } \\
\text { neurons }\end{array}$ & $\begin{array}{c}\text { Spread } \\
\text { constant }\end{array}$ & Target error \\
\hline 1 & Gaussian & 34 & 0.1 & $5 \times 10^{-3}$ \\
\hline 2 & Gaussian & 59 & 0.3 & $10^{-5}$ \\
\hline
\end{tabular}

In the experiments, the neural network systems are tested by activating with a certain temperature distribution over the slab at the time estimated by network 1 (such temperature distribution is chosen from one of the individual distributions that make up the one in Figure 3). The input data and the estimated time feed network 2 , for the iterative estimation of the initial distribution. Figure 5 shows a flowchart for the activation of both networks.

\section{Results}

Activation results are shown in Figure 6. The networks are presented to a certain temperature profile from one of the three individual distributions. The profiles supplied to the networks are chosen at four different instants of time corresponding to $1 / 2,1 / 5,1 / 10$, and $1 / 25$ of the total time for steady state.

The plots in Figure 6 show results with the activation of the backpropagation networks and the RBF networks for the same temperature distribution. For a better understanding, the curves with $\left(^{*}\right)$ represent the supplied temperature distribution. The curves with $(+)$ represent the target profile, that is, the desired initial temperature condition. The curves with (o) represent the neural network estimated initial condition. Figures 6-a e 6-b show the backpropagation and
RBF results for a triangular distribution chosen at $1 / 2$ of the time of steady state.

It is to be noticed that the multilayer perceptron with backpropagation approximates better to the desired profile. Figures 6-c and 6-d show the results for the temperature distribution measured at $1 / 5$ of steady state time. Figures $6-e$ and 6-f show the results for a logarithmic distribution chosen at $1 / 25$ of the steady state time with $5 \%$ noise added. Figures 6-g and 6-h show the results for the multilayer perceptron with backpropagation and the RBF networks for a distribution, which is not in the training phase. Comparisons show the multilayer perceptron with backpropagation networks generalize better than RBF networks for this application. Table 3 presents a summary of the described activation results.

Figure 7 shows the results for the multilayer perceptron neural network activation for a transient temperature distribution at $1 / 8$ of steady state time from the initial temperature profile. The sum of squared error was 0.0139 . A very small error is achieved, but a glance at Figure 7, reveals the poor quality of the solution.

(a)

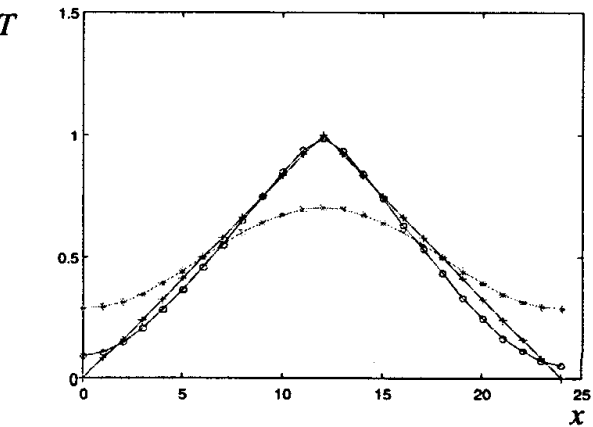

(b)

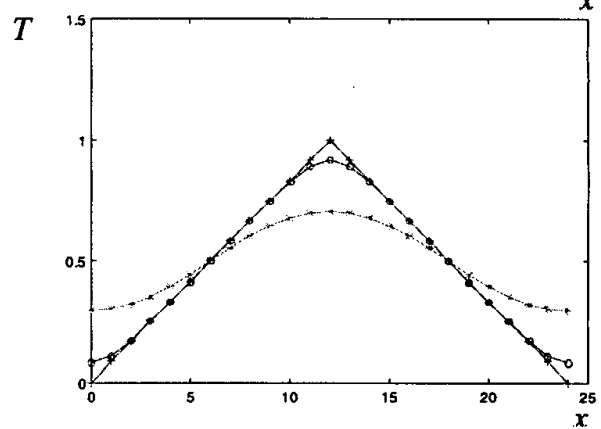

(c)

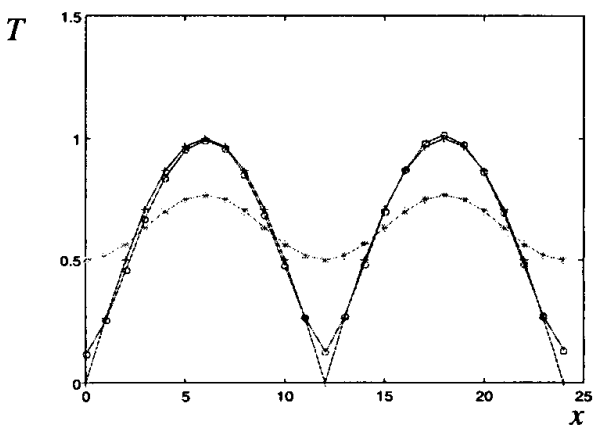


(d)

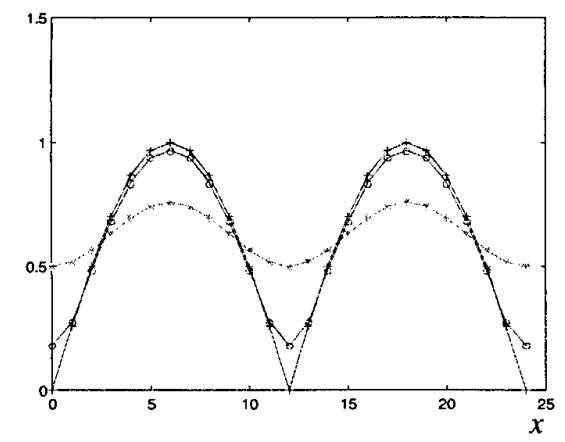

(e)

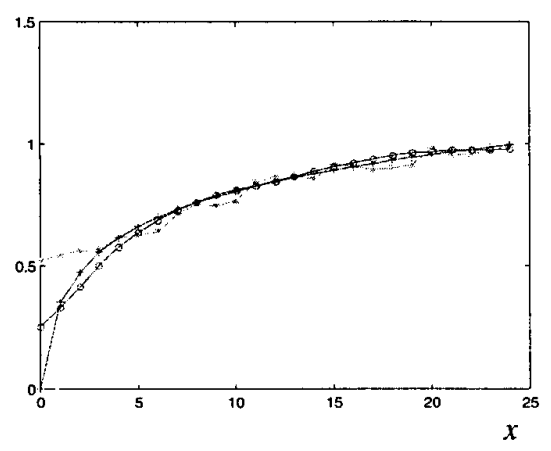

(f)

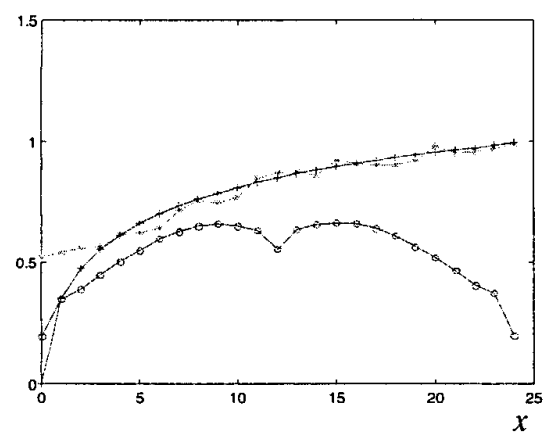

(g)

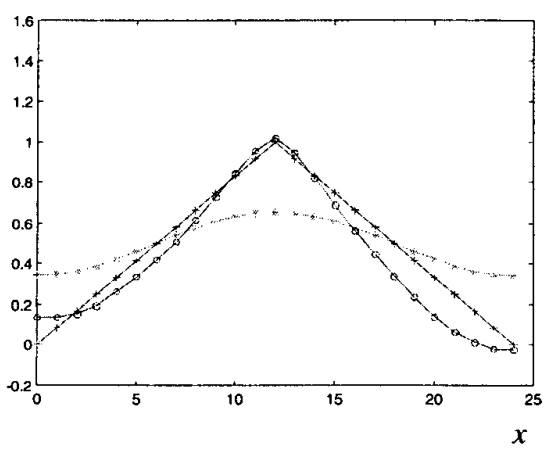

(h)

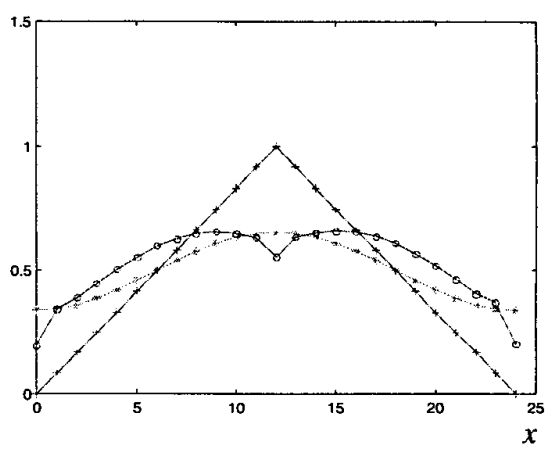

Figure 6 - Results of activation of both network arrangements.

\section{Conclusions}

In this paper an inverse initial condition problem in heat conduction is approached by using neural network systems. Two neural network architectures are used: the multilayer perceptron with backpropagation and radial basis function. The training sets are constructed by applying the direct model in equation 2 to three different initial condition. The two neural networks systems are composed of two multilayer perceptron and two radial basis function networks.

Figure 6 and Table 3 show the multilayer perceptron with backpropagation interpolates better for unknown data (Figure 6-g) and are more robust to perturbations in the data (Figure 6-e). Radial Basis Function networks train faster but are very sensitive to noise (figure 6-h). Figure 7 shows that neural networks require further training and investigation to improve its generalization capacity.

Although the results presented in this paper prove the effectiveness of neural networks for solving inverse problems. On going work is being conducted to improve the performance of the solutions and to try different neural network architectures, such as the cascade correlation neural network. Also, there exist many different inverse problems in several application areas in which neural network systems shall be tried, for instance in geophysics, image processing, and computer vision.

Table 3 - Summary of activation results

\begin{tabular}{|l|l|l|l|l|}
\hline Distribution & Time & $\begin{array}{l}\text { Error } \\
\text { Back }\end{array}$ & Error RBF & Noise \\
\hline Triangular & $1 / 2$ & 0.001932 & 0.000912 & $0 \%$ \\
\hline Triangular & $1 / 5$ & 0.000573 & 0.000425 & $0 \%$ \\
\hline Triangular & $1 / 25$ & 0.000706 & 0.000408 & $0 \%$ \\
\hline Sinusoidal & $1 / 5$ & 0.002148 & 0.004418 & $0 \%$ \\
\hline Logarithmic & $1 / 25$ & 0.003241 & 0.001665 & $0 \%$ \\
\hline Triangular & $1 / 5$ & 0.001311 & 0.000465 & $5 \%$ \\
\hline Logarithmic & $1 / 2$ & 0.002974 & 0.102762 & $5 \%$ \\
\hline Triangular & $3 / 10$ & 0.009997 & 0.039955 & $0 \%$ \\
\hline
\end{tabular}




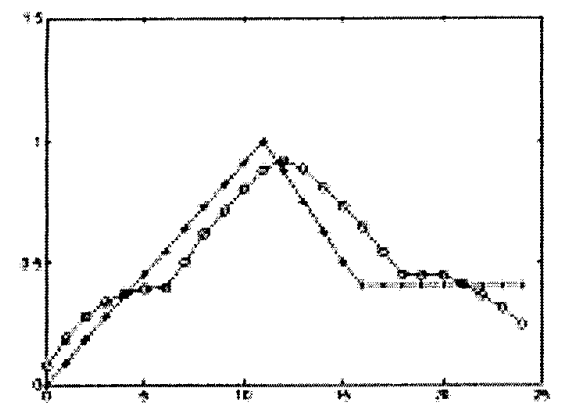

Figure 7 - Initial condition estimated from unknown temperature distribution.

\section{Acknowledgements}

This work has been supported by FAPESP, São Paulo State Foundation for Research Support.

\section{References}

[1] Haykin, S., Neural Networks Expand SP's Horizons. IEEE Signal Processing, March 1996, pp. $24-49$.

[2] Widrow, B.; Winter, R., Neural Nets for Adaptive Filtering and Adaptive Pattern Recognition. Computer, 21(3), March 1988, pp. 25-39.

[3] Krejsa,J.; Woodbury, K.A.; Ratliff, J.D.; Raudensky, M., Assessment of Strategies and Potential for Neural Networks in the IHCP, Inverse Problems in Engineering, 7(3), 197-213.

[4] Muniz, W.B.; Campos Velho, H.F.; Ramos, F.M., A Comparison of Some Inverse Methods for Estimating the Initial Condition of the Heat Equation. Journal of Computational and Applied Mathematics, Vol. 101, pp. 153-171, 1999

[5] Musavi, M.T.; Ahmed, W.; Chan, K.H.; Faris, J.B.; Hummels, D.M., On the Training of Radial Basis
Function Classifiers. Neural Networks, Vol. 5, pp. 595603, 1992.

[6] Chen, S.; Cowan, C.F.N.; Grant, P.M., Orthogonal Least Squares Learning Algorithm for Radial Basis Function Networks. IEEE Transactions on Neural Networks, vol. 2, No. 2, pp. 302-309, March 1991.

[7] Mulgrew, B., Applying Radial Basis Functions. IEEE Signal Processing Magazine, pp. 50-65, 1996.

[8] Coccorese, E.; Martone, R.; Morabito, C. (1994): A Neural Network Approach for the Solution of Electric and Magnetic Inverse Problems, IEEE Transactions on Magnetics, 30(5), 2829-2838.

[9] Lin, C-T; Lee, G. Neural Fuzzy Systems: A Neuro-Fuzzy synergism to Intelligent Systems. Prentice Hall, New Jersey, 1996

[10] Tsoukalas, L.H.; Uhrig, R.E., Fuzzy and Neural Approaches in Engineering. John Wiley, New York, 1997.

[11] Haykin, S. Neural Networks: A Comprehensive Foundation. Macmillan, New York, 1999.

[12] Nadler, M.; Smith, E.P., Pattern Recognition Engineering. John Wiley, New York, 1993

[13] N.J. McCormick, Inverse Problems: Methods and Applications, TEMA: Tendências em Matemática Aplicada e Computacional, 2(2001), 1-12

[14] Elshafiey, I.; Udpa, L.; Udpa, S., (1995): Solution of Inverse Problems in Electromagnetics Using Hopfield Neural Networks, IEEE Transactions on Magnetics, 31(1), 852-861.

[15] Hidalgo, H.; Gómez-Treviño, E., (1996): Application of Constructive Learning Algorithms to the Inverse Problem, IEEE Transactions on Geoscience and Remote Sensing, 34(4), 874-885.

[16] Silva, J.D.S.; Mikki, F.T.; Issamoto, E.; Luz, J.I.; Oliveira, P.P.B.; Campos-Velho, H.F., An inverse initial condition problem in heat conduction: a neural network approach. In: XV Congresso Brasileiro de Engenharia Mecânica - COBEM, 1999, Águas de Lindóia. 1999. v.1. 